\title{
A study of the remarkable galaxy system AM 546-324 (the core of Abell S0546) ${ }^{\star}$
}

\author{
M. Faúndez-Abans ${ }^{1}$, A. C. Krabbe ${ }^{2}$, M. de Oliveira-Abans ${ }^{1,3}$, P. C. da Rocha-Poppe ${ }^{4,5}$, I. Rodrigues ${ }^{2}$, \\ V. A. Fernandes-Martin ${ }^{4,5}$, and I. F. Fernandes ${ }^{4,5}$

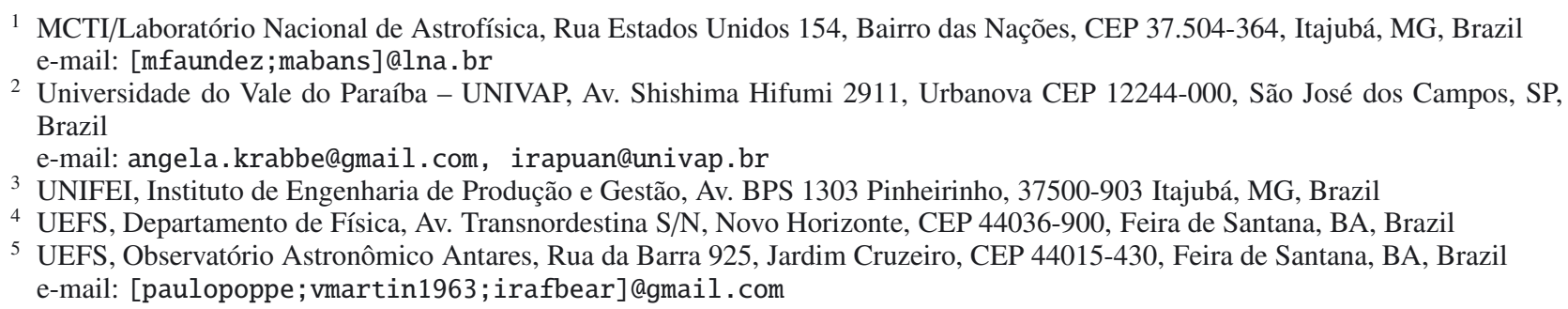

Received 17 February 2012 / Accepted 7 May 2012

\begin{abstract}
Aims. We report first results of an investigation of the tidally disturbed galaxy system AM 546-324, whose two principal galaxies 2MFGC 04711 and AM 0546-324 (NED02) were previously classified as interacting doubles. This system was selected to study the interaction of ellipticals in a moderately dense environment. We provide spectral characteristics of the system and present an observational study of the interaction effects on the morphology, kinematics, and stellar population of these galaxies.

Methods. The study is based on long-slit spectrophotometric data in the range of $\sim 4500-8000 \AA$ obtained with the Gemini MultiObject Spetrograph at Gemini South (GMOS-S). We have used the stellar population synthesis code STARLIGHT to investigate the star formation history of these galaxies. The Gemini/GMOS-S direct r-G0303 broad band pointing image was used to enhance and study fine morphological structures. The main absorption lines in the spectra were used to determine the radial velocity.

Results. Along the whole long-slit signal, the spectra of the Shadowy galaxy (discovered by us), 2MFGC 04711, and AM 0546-324 (NED02) resemble that of an early-type galaxy. We estimated redshifts of $z=0.0696, z=0.0693$ and $z=0.0718$, corresponding to heliocentric velocities of $20141 \mathrm{~km} \mathrm{~s}^{-1}, 20057 \mathrm{~km} \mathrm{~s}^{-1}$, and $20754 \mathrm{~km} \mathrm{~s}^{-1}$ for the Shadowy galaxy, 2MFGC 04711 and AM 0546-324 (NED02), respectively. The central regions of 2MFGC 04711 and AM 0546-324 (NED02) are completely dominated by an old stellar population of $2 \times 10^{9}<t \leq 13 \times 10^{9} \mathrm{yr}$ and do not show any spatial variation in the contribution of the stellar-population components.

Conclusions. The observed rotation profile distribution of 2MFGC 04711 and AM 0546-324 (NED02) can be adequately interpreted as an ongoing stage of interaction with the Shadowy galaxy as the center of the local gravitational potential-well of the system. The three galaxies are all early-type. The extended and smooth distribution of the material in the Shadowy galaxy is a good laboratory to study direct observational signatures of tidal friction in action.
\end{abstract}

Key words. galaxies: general - galaxies: interactions - galaxies: kinematics and dynamics - galaxies: individual: AM 0546-324 galaxies: individual: 2MFGC 04711

\section{Introduction}

Galaxy interactions and mergers are fundamentally important in the formation and evolution of galaxies. Hierarchical models of galaxy formation and various observational evidence suggest that elliptical galaxies are, like disk galaxies, embedded in massive dark-matter halos. Lenticular and elliptical galaxies, called early-type galaxies have been thought to be the end point of galaxy evolution. These systems have shown uniform red optical colors and display a tight red sequence in optical colormagnitude diagrams (e.g. Baldry et al. 2004). Their color separation from star-forming galaxies is thought to be due to a lack of fuel for star formation, which must have been consumed, destroyed or removed on a reasonably short timescale (e.g. Faber et al. 2007). In addition, numerical simulations have shown that

^ Based on observations made at the Gemini Observatory, under the identification number GS-2010B-Q-7. the global characteristics of the binary merger remnants of two equal-mass spiral galaxies, called major mergers, resemble those of early-type galaxies (Toomre \& Toomre 1972; Hernquist \& Barnes 1991; Barnes 1992; Mihos et al. 1995; Springel 2000; Naab \& Burkert 2003; Bournaud et al. 2005). Remnants with properties similar to early-type objects can also be recovered through a multiple minor merger process, the total accreted mass of which is at least half of the initial mass of the main progenitor (Weil \& Hernquist 1994, 1996; Bournaud et al. 2007). This scenario of early-type formation through accretion and merging of bodies would fit well within the frame of the hierarchical assembly of galaxies provided by cold dark matter cosmology.

Interactions between early-type galaxies are less spectacular than those observed in spiral galaxies. While impressive tidal tails, plumes, bridges, and shells are observed in tidally disturbed spirals, the effects of the interaction are less easily recognized in elliptical galaxies, since they have little gas and dust, and are 
Table 1. Basic properties of the principal galaxies of the system AM 0546-324.

\begin{tabular}{|c|c|c|c|c|}
\hline Parameter & 2MFGC 04711 & Shadowy galaxy & AM 0546-324 (NED02) & Ref. \\
\hline$\overline{\mathrm{RA}(2000)}$ & 054834.1 & 054834.7 & 054835.1 & this work \\
\hline $\operatorname{Dec}(2000)$ & -323930.9 & -323946.2 & -324001.0 & this work \\
\hline Morphological classification & Elliptical & $\mathrm{Cd} ?$ & Elliptical & this work \\
\hline$z$ & 0.0693 & 0.0696 & 0.0718 & this work \\
\hline$V\left(\mathrm{~km} \mathrm{~s}^{-1}\right)(a)$ & $20057 \pm 10$ & $20141 \pm 10$ & $20754 \pm 10$ & this work \\
\hline$V\left(\mathrm{~km} \mathrm{~s}^{-1}\right)(b)$ & $20793 \pm 10$ & $20880 \pm 10$ & $21526 \pm 10$ & this work \\
\hline$z$ & 0.0692 & & 0.0721 & $\operatorname{NED}(q)$ \\
\hline$V\left(\mathrm{~km} \mathrm{~s}^{-1}\right)$ & $20749 \pm 40$ & & $21615 \pm 26$ & $\operatorname{NED}(q)$ \\
\hline Magnitude & $15.0 \mathrm{R}$ & & & NED \\
\hline Other designations & 2MASX J05483415-3239306 & & 2MASX J05483518-3240006 & NED \\
\hline Distance (Mpc) & 292.6 & 293.8 & 303.0 & this work \\
\hline Distance (Mpc) & 293.0 & & 305.0 & NED \\
\hline$\sigma_{v}\left(\mathrm{~km} \mathrm{~s}^{-1}\right)$ & 312 & 365 & 197 & this work \\
\hline Mass (lower limit) & $1.63 \times 10^{11} M_{\odot}$ & $5.24 \times 10^{11} M_{\odot}$ & $1.60 \times 10^{11} M_{\odot}$ & this work \\
\hline U-shaped base $(r)$ & $2{ }^{\prime \prime} 0$ & & 1 '. 54 & this work \\
\hline Major axis (lower limit) $(r)$ & 9.42 & $21^{\prime \prime} .73$ & $8 . .53$ & this work \\
\hline$J-H$ & 0.404 & & 0.732 & NED \\
\hline$H-K$ & 0.142 & & -0.050 & NED \\
\hline$J-K$ & 0.546 & & 0.641 & NED \\
\hline
\end{tabular}

Notes. (a): extracted for high velocities (Lang 1999), see also Lindengren \& Dravins (2003); (b): non-relativistic velocity using the standard formula (Lang 1999); (r): measurements on the Gemini/GMOS-S r-G0303-filter; $(q)$ : original data from Quintana \& Ramírez (1995).

dominated essentially by old stellar populations. Evidence for recent merger-driven star formation (Rogers et al. 2009) and morphological disturbances such as shells, ripples, and rings have been observed in early-type galaxies (Kaviraj et al. 2010 and Wenderoth et al. 2011).

The peculiar Ring Galaxies (pRGs) show a wide variety of ring and bulge morphologies and were classified by Faúndez-Abans \& de Oliveira-Abans (1998) into five families, following the general behavior of galaxy-ring structures. From these categories eight morphological subdivisions are highlighted. One of these morphological subdivisions is a basic structure called Solitaire. The pRG Solitaire is described as an object with the bulge on the ring, or very close to it, resembling a one-diamond finger ring (single knotted ring). In these objects, the ring generally looks smooth and thinner on the opposite side of the bulge (as archetypes FM 18815/NED02, AM 0436-472/NED01), ESO 202-IG45/NED01 and ESO 303-IG11/NED01). Although the statistics are as yet poor, the Solitaire type is probably produced by the interaction between elliptical-like galaxies and/or gas-poor S0 galaxies with an elliptical companion. In a forthcoming paper, a list of Solitaire-type pRGs and a preliminary study and statistics will be presented.

There are no reports of Solitaires in early stages of formation in the literature yet; so a few pairs of galaxies were selected as early-stage candidates (see one of them in Wenderoth et al. 2011). Even though one of the selected candidates, AM 546-324, originally extracted from Arp and Madore's catalog (Arp \& Madore 1977, 1986; category 2, interacting doubles), seems to be morphologically different from an expected Solitaire in the early stage, it is remarkable enough to be studied as an almost isolated "spherical/elliptical and S0 interacting objects" in centrally sparse clusters of galaxies.

In this paper, we report new results for the tidally disturbed galaxy system AM 546-324 based on data obtained from longslit spectrophotometric observations at Gemini Observatory, in Chile. Values of $H_{\mathrm{o}}=70 \mathrm{~km} \mathrm{~s}^{-1} \mathrm{Mpc}^{-1}, \Omega_{\text {matter }}=0.27$ and $\Omega_{\text {vaccum }}=0.73$ have been adopted throughout this work (Freedman et al. 2001; Astier et al. 2006, and Spergel et al. 2003).

\section{AM 0546-324 review}

The existing information on this object comprises: (1) the Arp-Madore catalog (Arp \& Madore 1986) referred to it as "Category 2: interacting doubles", which are objects consisting of two galaxies that, by their apparent magnitude and spacing, appear to be associated; (2) the redshifts of 165 Southern rich Abell cluster of galaxies by Quintana \& Ramírez (1995), in which the galaxy system AM 546-324 is a member of Abell S0546; (3) the 2MASS-selected flat galaxy catalog by Mitronova et al. (2004); (4) the catalog of near-infrared properties of LEDA galaxies using the full-resolution images from the DENIS survey (Paturel et al. 2005); and (5) the entry from the 6dFGS-NVSS data by Mauch \& Sadler (2007). In Abell et al. (1989), S0546 is quoted as irregular following the cluster classification in Abell's (1965) system, with 23 cluster members. Using the data quoted in Abell et al. (1989) in a magnituderedshift relation for the Abell southern clusters, and using the S0546 distance class $m_{10}=5$ result in an approximate radial velocity of $c z=20893 \mathrm{~km} \mathrm{~s}^{-1}$, which agrees with our results (using the non-relativistic velocity formula, see Table 1).

Figure 1 displays the AM 546-324 system in a 5 minuteexposure GMOS-S pointing image in the $r$-G0303 filter (effective wavelength of $6300 \AA$ ). Table 1 displays the new velocity and $z$ values, together with some early information on the principal members of AM 0546-324, Table 2 displays some information on relevant objects in and around the system.

\section{Observations and data reduction}

The spectroscopic observations were performed with the 8.1-m Gemini South telescope (Chile) (ID program GS-2010BQ-7). We used the GMOS-S spectrograph in long-slit mode 
Table 2. Relevant objects in and around system AM 0546-324.

\begin{tabular}{lcccccc}
\hline \hline Object & RA $(2000)$ & Dec $(2000)$ & $z_{\text {abs }}$ & $V\left(\mathrm{~km} \mathrm{~s}^{-1}\right)$ & Distance $(\mathrm{Mpc})$ & Ref. \\
\hline J054832.5-323954.1 & 054832.5 & -323954.1 & & & & Polar Ring? this work \\
J054834.2-323944.5 & 054834.2 & -323944.5 & 0.0698 & 20923 & 298.9 & The Knot $(a)$ \\
J054835.4-324011.5 & 054835.4 & -324011.5 & 0.0685 & $20550 \pm 40$ & 293.6 & C, this work $(b)$ \\
\hline
\end{tabular}

Notes. (a) Quintana \& Ramírez (1995); (b) the compact companion C close to AM 0546-324 (NED02).

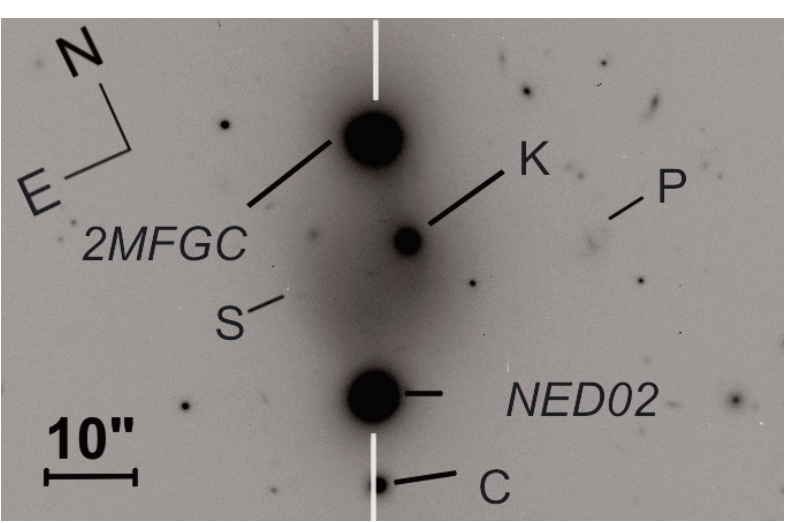

Fig. 1. System AM0546-324. Optical 5-min exposure GMOS-S image in the $r$-G0303 filter, enhanced by a median filter kernel of $300 \times$ 300 pixels (see Faúndez-Abans \& de Oliveira-Abans 1998 for details on the method). The slit position $\mathrm{PA}=157^{\circ}$ is also displayed as two short white lines to preserve the image of the objects. The letters stand for: $\mathrm{K}$, "the Knot" (Quintana \& Ramírez 1995); S, the Shadowy galaxy; C, a companion galaxy; and $\mathrm{P}$, a probable Polar Ring galaxy.

(Hook et al. 2004) ${ }^{1}$. The R400+G5325 grating was centered at $6250 \AA$ and used with a long-slit $1.5 \operatorname{arcsec} \times 375$ arcsec. The data were binned by 2 in the spatial dimension and 2 in the spectral dimension, producing a spectral resolution of $\sim 5.1 \AA$ FWHM, sampled at $0.68 \AA \mathrm{pix}^{-1}$. The seeing throughout the observations was 0.54 and the binned pixel scale was 0 .'145 $\mathrm{pix}^{-1}$. The wavelength range was $\sim 4500-8000 \AA$. The spectrophotometric standard star H 600 was observed using the same experimental set up. The long-slit spectra were taken at one position angle on the sky, $\mathrm{PA}=157^{\circ}$, to encompass the three objects in one shot, and it almost crossed the center of each object.

The standard Gemini-IRAF routines were used to carry out bias subtraction, flat-fielding, and cosmic ray subtraction. The data were then wavelength calibrated with an accuracy $\leqslant 0.3 \AA$. The binned 2D spectra were then flux-calibrated using the photometric standard star H600. The 2D spectra were then extracted into 1D spectra, which were sky-subtracted and binned in the spatial dimension. We have cross-correlated our observed spectra with three galaxy and star templates with good signal-to-noise. These results were checked with the composite absorption-line template "fabtemp97" distributed by $\mathrm{RVSAO}^{2} / \mathrm{IRAF}$ external package. We adopted the redshift value from the best highest correlated coefficient template.

The spectral apertures were extracted with the APALL/IRAF package and three methods: (1) the standard IRAF procedure; (2) overlapping a shifted sample with steps $<5^{\prime \prime}$, which causes

\footnotetext{
${ }_{1}$ A description of the instrument can be found at http://www .gemini.edu/sciops/instruments/gmos

2 The RVSAO IRAF (Radial Velocity Package for IRAF) external package was developed at the Smithsonian Astrophysical Observatory. Full documentation of this software, including numerous examples of its use, in on-line at http://tdc-www . harvard. edu/iraf/rvsao/
}

oversampling; (3) and the event-covering method, for which we used the aperture step as a mapping event process (Wong \& Chiu 1987³; see also Schafer 1997 and Wu \& Barbara 2002), which also causes oversampling. The idea of the last two procedures was to use the aperture size as a filter to detect kinematical structures in the long-slit velocity map. The results of the first two procedures have been used in this work. The third method was mainly used when the original data were either corrupted or incomplete. Its results were not different from those of the second method because of the data completeness.

\section{Analysis and results}

\subsection{The field around AM 0546-324}

As can be seen in Fig. 1, this system of galaxies is seen almost edge-on, with five prominent objects in the field. According to the estimated distances, they may be physically associated. In Fig. 1, from NW to SE, these objects are (1) the almost spherical galaxy 2MFGC 04711; (2) the galaxy "Knot", K, as labeled by Quintana \& Ramírez (1995); (3) the object that we have named the Shadowy galaxy (hereafter the $S$ galaxy), whose center is enhanced in the figure; (4) AM 0546-324 (NED02) (hereafter NED02), which is almost spherical; and (5) the compact anonymous spherical galaxy "C". Another relevant object to the SW in the field (better seen in Fig. 2, bottom panel) is a probable Polar Ring galaxy, named here "P", whose redshift value is not known yet.

To extract as much information as possible from the GMOS-S r-G0303 pointing image, we used different spatial filtering to find fine morphological structures in the frame. The top panel of Fig. 2 displays the result of applying a median filter kernel of $100 \times 100$ pixels, where the S galaxy appears elongated, its center and a few rims having also been enhanced. A few thin filaments in the $\mathrm{K}$ object, with one of them pointing to the center of the $\mathrm{S}$ galaxy, have also been enhanced. Furthermore, the galaxies K, 2MFGC 04711, and NED02 appear to be slightly deformed by the tidal interaction. The lower panel of Fig. 2 displays a median filter kernel of $500 \times 500$ pixels where the deformation of the main objects and some faint dwarf structures apparently bound to this system have been enhanced. The rims and the center of $S$ galaxy are still evident. The upper panel of Fig. 3 shows the center and the rims of the S galaxy, after a lowpass filter was used. It highlights the deformation of the elliptical galaxies and enhances a few notable dwarf satellites around this system. The bottom panel of Fig. 3 is a zoom on the $\mathrm{S}$ galaxy to better illustrate its center and a few very faint rims, after using a Gaussian filter.

To determine the central ellipticity of the galaxies, we used the ELLIPSE STSDAS-task ${ }^{4}$, which fits elliptical isophotes to

\footnotetext{
3 The event-covering method is defined as a strategy of selecting a subset of statistically independent events in a set of variable-pairs, regardless of their statistical independence.

4 Space Telescope Science Data Analysis Software Package.
} 


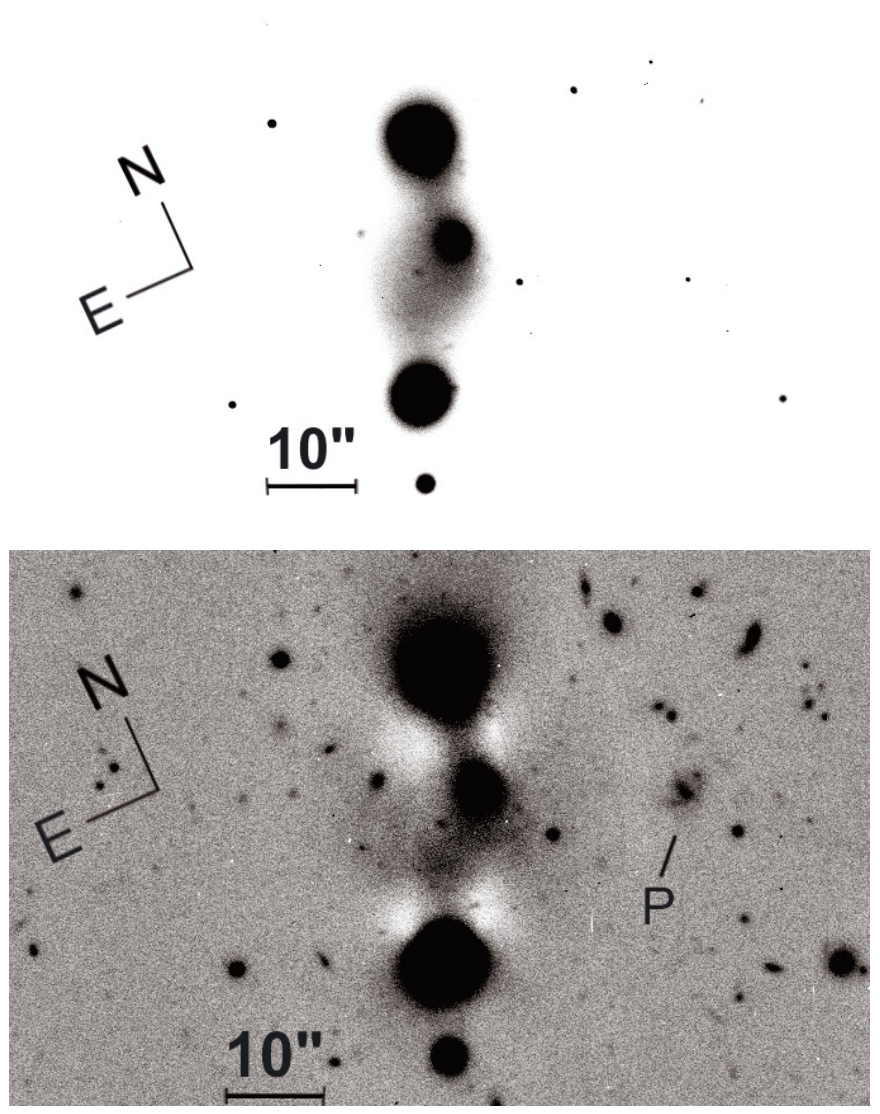

Fig. 2. From top to bottom: a) median filtering with a $100 \times 100$-pixel kernel after the original image subtraction, b) same as first panel for a $500 \times 500$-pixel kernel. The clear patches are artifacts of the method, but the fine structures are preserved. The candidate for a Polar Ring, which has been discovered in this work, is marked by the letter "P".

galaxy direct images. Then we created a 2D noiseless model image using the BMODEL STSDAS-task built from the results of the isophotal analysis. Table 3 lists rough estimates of the "non perturbed" elliptical-class section and the whole major axisdiameter in kpc for the quoted galaxies.

\subsection{The spectra and kinematics}

We report the first dedicated long-slit spectroscopic results for the three main galaxies of the AM 0546-324 system. A sample of the spectra of these galaxies are shown in Fig. 4. The $\lambda \lambda 5000-7750 \AA$ spectral section of 2MFGC 04711 and NED02 are displayed, while the S galaxy's is displayed only in the $\lambda \lambda 5000-5800 \AA$ spectral section. They show the main stellar absorption features identified in the nuclear region: $\mathrm{H} \beta, \mathrm{MgIb} \lambda 5174, \mathrm{MgH} \lambda 5269$, NaID $\lambda 5892$, the TiO bands $\lambda \lambda 6250,7060$, and the $\mathrm{O}_{2}$ atmospheric band. The column density and positions of these lines were determined by fitting a Gaussian to the observed profile. This procedure was reviewed using the RVSAO/XCSAO package. The spectra in the regions $\lambda \lambda 7750-8000 \AA$ (for 2MFGC 04711 and NED02) and $\lambda \lambda 5800-8000 \AA$ (for the S galaxy) were not taken into account because of the high noise. The characteristics of the spectra are discussed below.

\subsection{1. $2 \mathrm{MFGC} 04711$}

No emission lines were detected along the slit. The spectral profile resembles that of an early-type galaxy. Using the NaID,
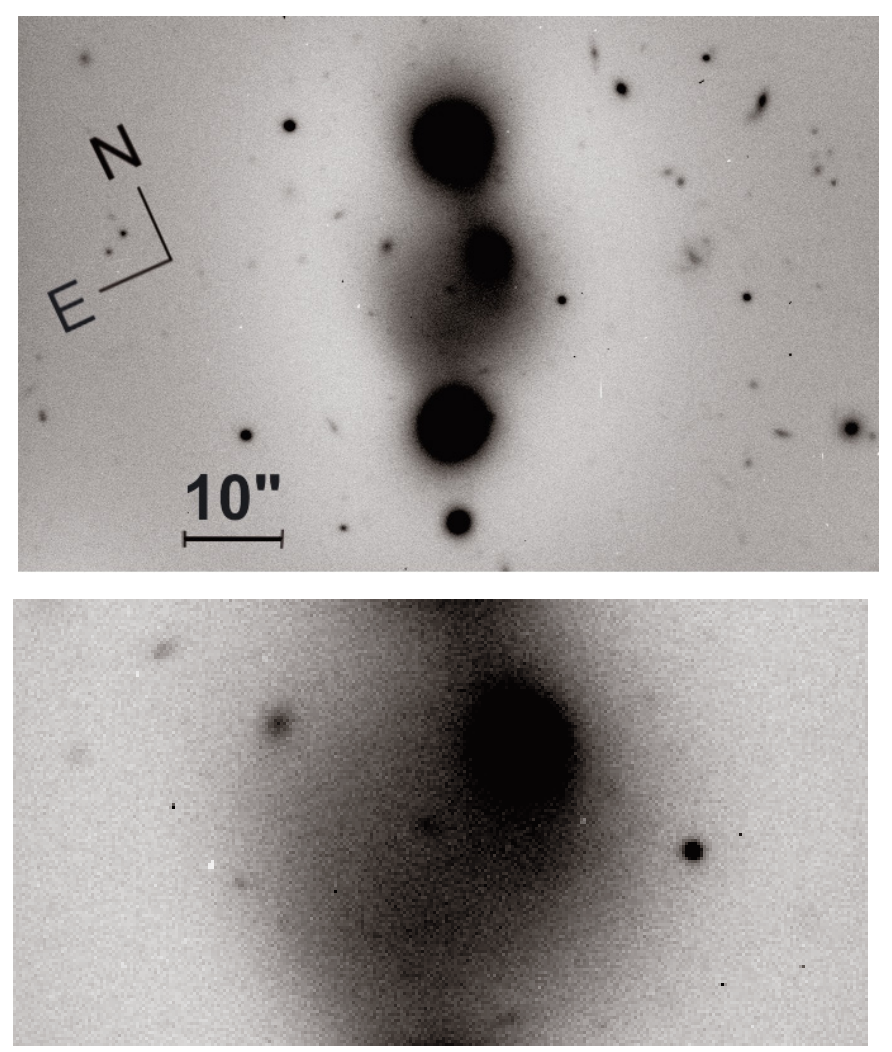

Fig. 3. From top to bottom: a) resulting image after using a low-pass filter (see text), b) same as first panel for a Gauss filter, zooming on the S galaxy.

Table 3. Ellipticity and major axis diameter.

\begin{tabular}{lcc}
\hline \hline Object & $\begin{array}{c}\text { "Bulge-like" section } \\
\text { (elliptical class) }\end{array}$ & $\begin{array}{c}\text { Major diameter } \\
(\mathrm{kpc})\end{array}$ \\
\hline 2MFGC 04711 & E1 & 13.7 \\
Shadowy & E3/4:: & $31.6:$ \\
NED02 & E0 & 12.4 \\
The Knot & E1: & 9.5 \\
C companion & E1 & 3.6 \\
\hline
\end{tabular}

$\mathrm{MgIb}$, and $\mathrm{H} \beta$ absorption lines, we cross-correlated our observed spectra with templates with good signal-to-noise and these results were checked with the composite absorption-line template "fabtemp97" distributed by RVSAO. We derived $z=0.0693$ and a heliocentric radial velocity of $V=20057 \pm 10 \mathrm{~km} \mathrm{~s}^{-1}$. The velocity dispersion $\sigma_{v}$ values were estimated by crosscorrelation with $\mathrm{K}$ and $\mathrm{M}$ star templates, with statistical error in $\sigma_{v}$ in the range of $10 \%-15 \%$ (XCSAO/RVSAO, also tested with XCOR/STSDAS). The dynamical masses were estimated based on a virial relation, the effective radius and the velocity dispersion of each galaxy. The derived effective radius and dynamical mass fit within $\leq 1 \sigma$ with the size-mass relation presented by Bezanson et al. (2011), predicts values close to those derived here for each galaxy. The calculated distance and dynamical mass are $292.6 \mathrm{Mpc}$ and $1.63 \times 10^{11} M_{\odot}$, respectively. Figure 5 displays the 2MFGC 04711 distribution of radial velocities measured from the $\mathrm{H} \beta, \mathrm{MgIb}$ and NaID absorption lines, along the slit-section (upper panel) and central region (lower panel). The errors of the individual velocity measurements do not exceed $10 \mathrm{~km} \mathrm{~s}^{-1}$ in the central region and increase to $20-40 \mathrm{~km} \mathrm{~s}^{-1}$ on its periphery. 

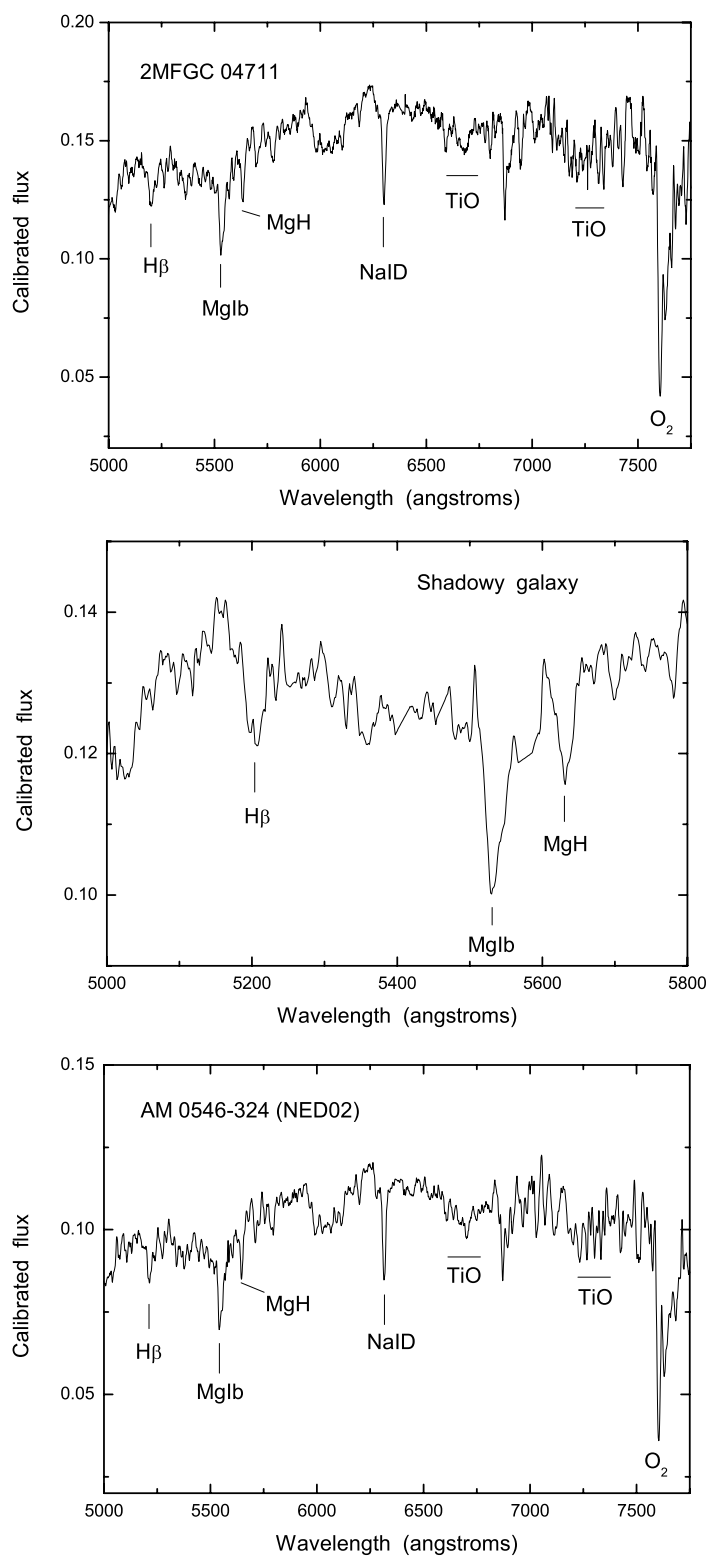

Fig. 4. Optical nuclear absorption features of 2MFGC 04711, the Shadowy galaxy and AM 0546-324 (NED02), in the upper, middle and lower panels, respectively. The spectra are displayed in units of $\times 10^{-16} \mathrm{erg} \mathrm{s}^{-1} \mathrm{~cm}^{-2} \AA^{-1}$.

\subsubsection{AM 0546-324 (NED02)}

NED02 also shows an early-type spectral profile. We estimated $z=0.0718$ and a heliocentric radial velocity of $V=$ $20754 \pm 10 \mathrm{~km} \mathrm{~s}^{-1}$. The calculated distance and dynamical mass are $303 \mathrm{Mpc}$ and $1.60 \times 10^{11} M_{\odot}$, respectively. Figure 6 displays the NED02 distribution of radial velocities measured from the MgIb and NaID absorption lines, along the slit-section (upper panel) and central region (lower panel). For clarity, the $\mathrm{H} \beta$ data are not shown in the first panel because they are mostly coincident with the NaID points at $r> \pm 1^{\prime \prime}$. The errors of the individual velocity measurements do not exceed $10 \mathrm{~km} \mathrm{~s}^{-1}$ in the central region and increase to $20-40 \mathrm{~km} \mathrm{~s}^{-1}$ on its periphery.

\subsubsection{The Shadowy galaxy}

We estimated $z=0.0696$ and a heliocentric radial velocity of $V=20141 \pm 10 \mathrm{~km} \mathrm{~s}^{-1}$, from an integrated central
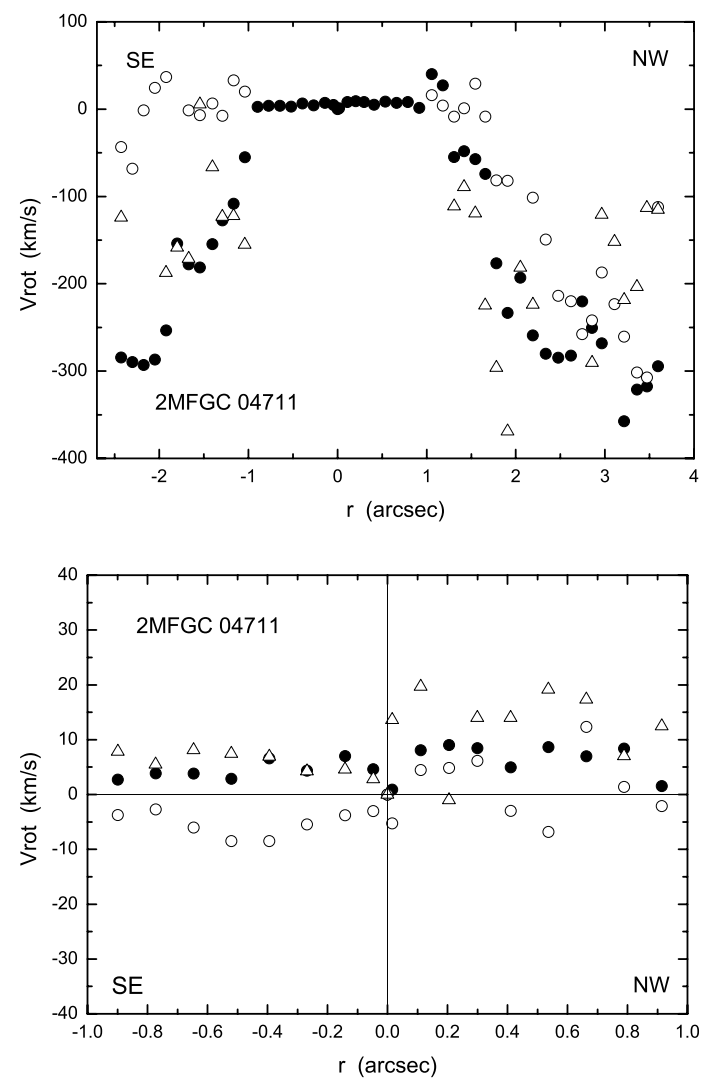

Fig. 5. Upper panel: the U-shaped rotation profile of $2 \mathrm{MFGC} 04711$ along the nucleus and bulge in the total observed long-slit $-3^{\prime \prime}$ to $+4^{\prime \prime}$ distribution (filled circles are data from NaID lines, open circles from $\mathrm{MgIb}$ and open triangles stand for $\mathrm{H} \beta$ lines); lower panel: same as first panel, for the central $-1^{\prime \prime}$ to $+1^{\prime \prime}$ core features, but using a shifted aperture sample with steps $<2$ " according to method (2) cited in Sect. 3.

spectral-section of 3 ".5. On the other hand, the calculated velocities for the NW and SE sections of the galaxy are $20193 \pm$ $20 \mathrm{~km} \mathrm{~s}^{-1}$ (NW) and $20081 \pm 20 \mathrm{~km} \mathrm{~s}^{-1}$ (SE), respectively (integrated NW-SE spectral-section of 6.' 1). The calculated distance and lower limit of the dynamical mass are $293.8 \mathrm{Mpc}$ and $5.24 \times 10^{11} M_{\odot}$, respectively.

The distribution of the radial velocities of 2MFGC 04711 and NED02 shows the U-shaped rotation profile (first panel of Figs. 5 and 6). This shape has been reported in studies of interacting binary-disturbed elliptical galaxies (see Borne 1990; Borne \& Hoessel 1985, 1985; Bender et al. 1991; Madejsky 1991 and Madejsky et al. 1991). The U-shaped profiles are common in strongly interacting elliptical galaxies, and the physical interpretation, given by Borne et al. (1994) is that there is a tidal coupling between the orbit of the companion and the resonant prograde rotating stars in the kinematically disturbed galaxy (Borne 1988; Borne \& Hoessel 1985; Bacells et al. 1989). The coupling of NED02 and 2MFGC 04711 with the $\mathrm{S}$ galaxy and the U-shaped rotation profile of these galaxies are thus a direct observational signature of tidal friction in action within this system, in agreement with the physical interpretation of Borne et al. (1994). Based on the merging times of simulations performed for a low-mass galaxy falling on to a massive elliptical by Leeuwin \& Combes (1997) and adopting for the $\mathrm{S}$ galaxy a $r_{\max }=30 \mathrm{kpc}$, our rough estimate for the decay times for 2MFGC 04711, NED02 and C galaxy are $4 \times 10^{8} \mathrm{yr}, 3.6 \times 10^{8} \mathrm{yr}$, and $5.6 \times 10^{8} \mathrm{yr}$, respectively. With the 

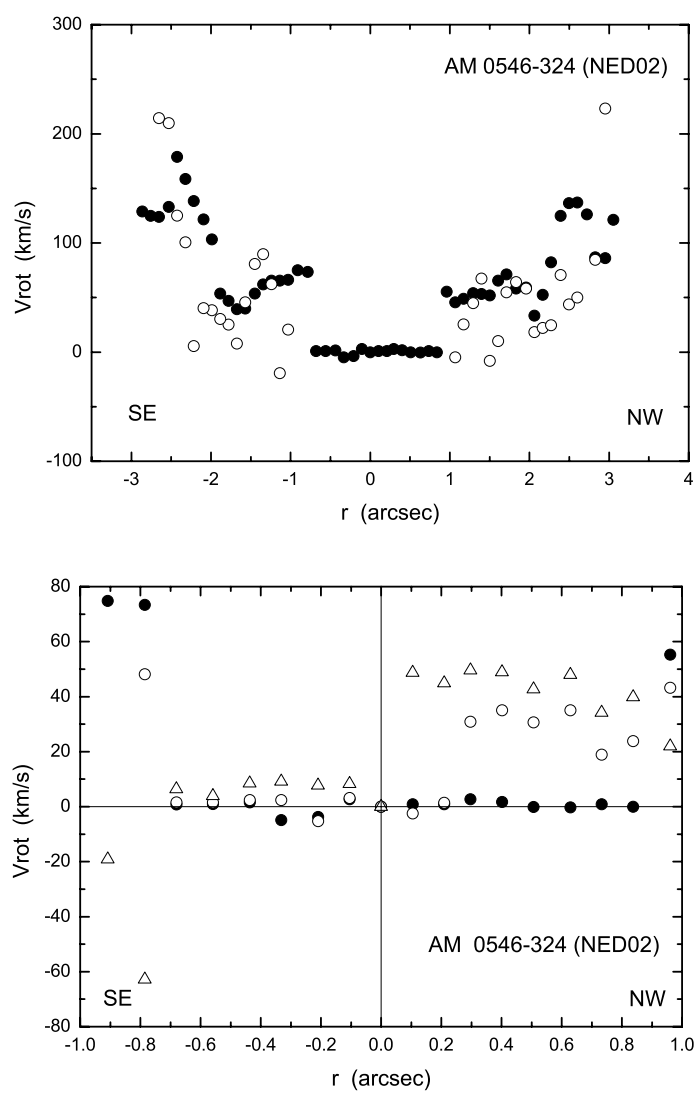

Fig. 6. From top to bottom: a) the U-shaped rotation profile of AM 0546-324 (NED02) along the nucleus and bulge in the total observed slit length $-3^{\prime \prime}$ to $+4^{\prime \prime}$ (filled circles are data from NaID lines, open circles from $\mathrm{MgIb}$ ); b) same as first panel, for the central $-1^{\prime \prime}$ to $+1^{\prime \prime}$ core features, but using a shifted aperture sample with steps $<2$ " according to method (2) cited in Sect. 3.

suitably simple model reported by Leeuwin \& Combes (1997) the amount of friction should accelerate in the decay time, which could be the scenario for the $\mathrm{S}$ satellite with a decay time shorter than $1.0 \times 10^{9} \mathrm{yrs}$.

The errors of the individual velocity measurements do not exceed $10 \mathrm{~km} \mathrm{~s}^{-1}$ in the central region of the galaxies and increase to $20-40 \mathrm{~km} \mathrm{~s}^{-1}$ at their periphery. There is a significant dispersion in the radial velocity distribution of both 2 MFGC and NED02 (see first panels of Figs. 5 and 6, respectively). The velocity spread is $\pm 40-110 \mathrm{~km} \mathrm{~s}^{-1}$ around $\pm 1^{\prime \prime}-3^{\prime \prime}$.

\subsection{Stellar population synthesis}

The detailed study of star formation in tidally perturbed galaxies provides important information not only on the age distribution of the stellar population, but also helps to better understand several aspects related to the interacting process and its effects on the properties of the individual galaxies and their evolution.

To investigate the star formation history of NED02 and 2MFGC, we used the stellar population synthesis code STARLiGHT (Cid Fernandes et al. 2004, 2005; Asari et al. 2007). This code has been extensively discussed in Cid Fernandes et al. (2004, 2005) and is built upon computational techniques originally developed for empirical population synthesis with additional ingredients from evolutionary synthesis models. This method was also used by Krabbe et al. (2011) and has been

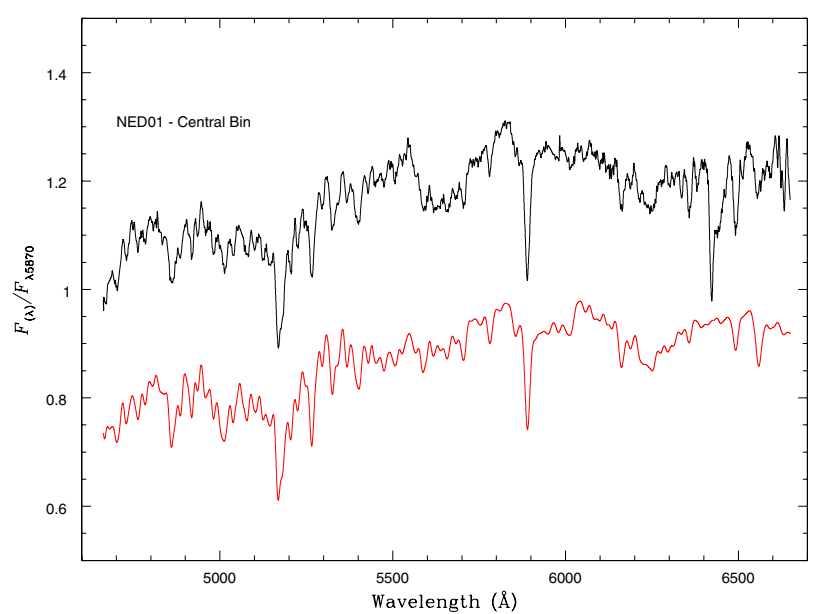

Fig. 7. Stellar population synthesis for $2 \mathrm{MFGC} 04711$. Central bin observed spectrum corrected for reddening (in black, shifted up by a constant) and the synthesized spectrum, in red.

successful in describing the stellar population in interacting galaxies.

The code fits an observed spectrum $O_{\lambda}$ with a combination of $N_{\star}$ single stellar populations (SSPs) from the Bruzual $\&$ Charlot (2003) models. These models are based on a highresolution library of observed stellar spectra, which allows for detailed spectral evolution of the SSPs at a resolution of $3 \AA$ across the wavelength range of 3200-9500 $\AA$ with a wide range of metallicities. We used the Padova 1994 tracks as recommended by Bruzual \& Charlot (2003), with the initial mass function of Salpeter (1955) between 0.1 and $100 M_{\odot}$. Extinction is modeled by STARLIGHT as due to foreground dust, using the Large Magellanic Cloud average reddening law of Gordon et al. (2003) with $R_{V}=3.1$, and parametrized by the $V$-band extinction $A_{V}$. The SSPs used in this work cover 15 ages namely, $t=0.001$, $0.003,0.005,0.01,0.025,0.04,0.1,0.3,0.6,0.9,1.4,2.5,5,11$, and $13 \mathrm{Gyr}$, as well as three metallicities, $Z=0.2 Z_{\odot}, 1 Z_{\odot}$, and $2.5 Z_{\odot}$, adding to $45 \mathrm{SSP}$ components. The fitting is carried out using a simulated annealing plus Metropolis scheme, with bad pixel regions excluded from the analysis.

Prior to the modeling, the SSPs models were convolved to the same resolution of the observed spectra; the observed spectra were shifted to their rest-frame, corrected for foreground Galactic reddening of $E(B-V)=0.036$ mag taken from Schlegel et al. (1998) and normalized to $\lambda 5870 \AA$. The error in $O_{\lambda}$ considered in the fitting was the continuum rms with a $S / N \geq 10$, where $S / N$ is the signal-to-noise ratio per $\AA$ in the region around $\lambda_{0}=5870 \AA$. In addition, the fitting was performed only in spectra with absorption lines.

Figures 7 and 8 show an example of the observed spectrum corrected by reddening and the model stellar population spectrum for 2MFGC 04711 and AM 0546-324 (NED02) galaxies, respectively. The results of the synthesis to the very central region are summarized in Table 4 for the individual spatial bins in each galaxy, stated as the perceptual contribution of each base element to the flux at $\lambda 5870 \AA$. Following the prescription of Cid Fernandes et al. (2005), we have defined a condensed population vector by binning the stellar populations according to the flux contributions into young, $x_{\mathrm{Y}}\left(t \leq 5 \times 10^{7} \mathrm{yr}\right)$; intermediateage, $x_{\mathrm{I}}\left(5 \times 10^{7}<t \leq 2 \times 10^{9} \mathrm{yr}\right)$; and old, $x_{\mathrm{O}}\left(2 \times 10^{9}<\right.$ $\left.t \leq 13 \times 10^{9} \mathrm{yr}\right)$ components. The same bins were used to represent the mass components of the population vector $m_{\mathrm{Y}}, m_{\mathrm{I}}$, 


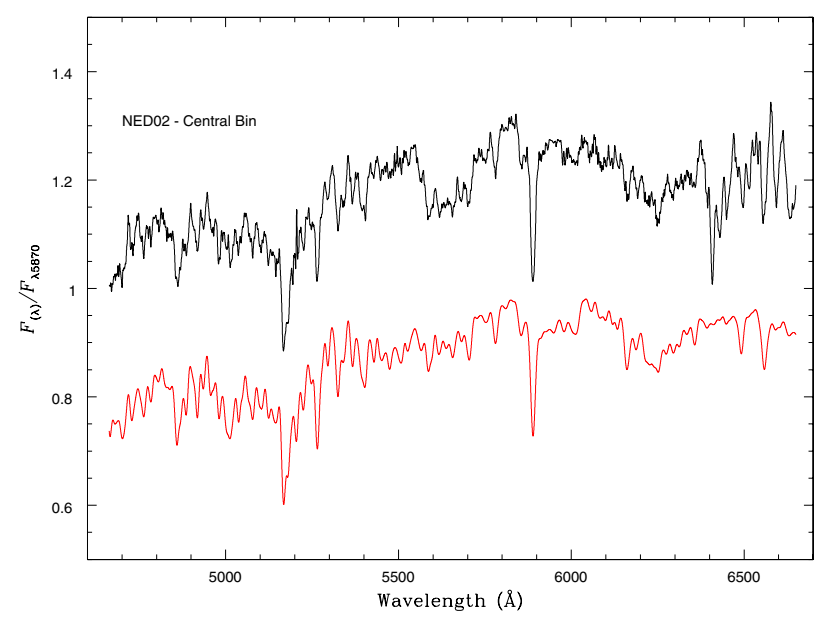

Fig. 8. Stellar population synthesis for NED02. Central bin observed spectrum corrected for reddening (in black, shifted up by a constant) and the synthesized spectrum, in red.

and $m_{\mathrm{O}}$ ). The metallicity $(Z)$, one important parameter to characterize the stellar population content, is weighted by light fraction. The quality of the fitting result is measured by the parameters $\chi^{2}$ and adev. The latter gives the perceptual mean deviation $\left|O_{\lambda}-M_{\lambda}\right| / O_{\lambda}$ over all fitted pixels, where $O_{\lambda}$ and $M_{\lambda}$ are the observed and model spectra, respectively.

The spatial variation in the contribution of the stellar population components for 2MFGC. NED02 is completely dominated by an old stellar population.

\section{Discussion}

The galaxy cluster Abell S0546 has 23 cluster members between $m_{3}$ and $m_{3}+2$ (Abell 1965), and the whole AM 0546-324 system is the core of the S0546. There are quite a few dwarf satellites around it, as expected for a local gravitational potential well defined by the AM 0546-324 system (see lower panel of Figs. 2 and 9). The main galaxies quoted in the literature are 2MFGC 04711, NED02, and the knot (K), which is described as an almost E1 galaxy (Quintana \& Ramírez 1995). Other members quoted in our study are the $\mathrm{S}$ galaxy and the $\mathrm{C}$ companion, which have been confirmed as members of this cluster. The derived radial velocity of the $\mathrm{S}$ galaxy is $20141 \mathrm{~km} \mathrm{~s}^{-1}$, which is our estimate of the radial velocity of the Abell S0546 core. The derived radial velocity of $\mathrm{S}$ using the non-relativistic formula agrees with the S0546 distance class $m_{10}=5(\mathrm{cz}=$ $20893 \mathrm{~km} \mathrm{~s}^{-1}$ ) extracted from Abell et al. (1989). Figure 10 displays the spatial distribution of the main galaxies of the AM 0546-324 system centered on the $S$ galaxy (see also Fig. 11).

Along the whole slit, the spectra of the three main galaxies show absorption lines characteristic of early-type objects. No star-forming regions and no nuclear ionization sources were detected. The whole AM0546-324 system seems to be tidally bound with radial velocity differences that range between 56-613 $\mathrm{km} \mathrm{s}^{-1}$. Adopting the $\mathrm{S}$ galaxy as the center of the system, the pair-velocity-difference combinations of the main objects are displayed in Table 5 . The nearby $\mathrm{C}$ galaxy was partially inside the GMOS-S slit and the estimated redshift $z=0.0685$ corresponds to a heliocentric radial velocity of $V=19834 \pm 30 \mathrm{~km} \mathrm{~s}^{-1}$, with a calculated distance of $289.2 \mathrm{Mpc}$ and a dynamical mass of $3.74 \times 10^{10} M_{\odot}$. The radial velocity of the $\mathrm{K}$ galaxy $\left(20923 \mathrm{~km} \mathrm{~s}^{-1}\right)$ was extracted from
Quintana \& Ramírez (1995). From the quoted mass, 2MFGC and NED02 each have approximately $31 \%$ of the mass of the $\mathrm{S}$ galaxy, and the $\mathrm{C}$ galaxy has almost $7 \%$. Evidence of the tidal interaction of this system are seen in the external deformations of 2MFGC 04711, NED02, and K galaxies, as well as the rims in the S galaxy (displayed in Figs. 1-3).

In this section, we propose the following kinematical behavior for the AM 0546-324 system based on spectroscopy and the direct image: (A) the S-galaxy is the center of the system, its SE-section is approaching and the NW-section is receding from us; (B) the 2MFGC 04711 galaxy is approaching us, and seems to be embedded in the peripheral material of the S-galaxy, its motion is retrograde from de S-galaxy apparent rotation; (C) NED02 is receding from us and its motion is also retrograde from the S-galaxy apparent rotation; (D) the K-galaxy is receding from us, and seems to be embedded in S-galaxy material and coupled prograde with the S-galaxy motion; (E) the C-galaxy is approaching us and coupled prograde with the S-galaxy motion.

Both 2MFGC 04711 and NED02 show a U-shaped rotation profile. In addition to the argument that tidal coupling in ellipticals with no net rotation will result in a U-shaped rotation profile with the galaxy core at the base of the U (Borne \& Hoessel 1985; Borne et al. 1994), it was also suggested that when the galaxy has a low degree of internal rotation, the tidal coupling should produce this U-type profile (Borne et al. 1994). The lower panels of Figs. 5 and 6 show the rotation profile in the interval $\pm 1^{\prime \prime}$ for the 2MFGC 04711 and NED02, respectively. The oversampled data are displayed in both figures to show signatures around the kinematical center. These figures suggest that (1) 2MFGC 04711 seems to have a low degree of perturbation and there is maybe a very slow internal rotation, its kinematical center is almost centered at the U-shaped-base profile; (2) NED02 seems to show no internal rotation in its U-shaped profile, but there is a break indicated by the $\mathrm{MgIb}$ and $\mathrm{H} \beta$ sampled data, for which we do not have a reliable explanation. The kinematical center is offcentered a few pc to the SE-direction in its U-shaped-base profile. This phenomenon is also seen in other pair interaction of Solitaire-type galaxies (see Faúndez-Abans et al. 2010).

Figures 2 (lower panel) and 9 show some dwarf objects around the AM 0546-324 system. A few of those objects may be aligned with the visible AM 0546-324 structure. This sparse system has an intrinsic tidal field, which could be an interesting laboratory for studying the relationship between the central components and the dark matter halo in weak fields (see simulations for a denser cluster environment by Pereira \& Bryan 2010). A non-negligible anonymous galaxy is to the east between the field galaxy 2MASX J05481766-3239441 and the AM 0546-324 system. The coordinates of the centroid (J2000), as calculated differentially from the centroid of 2MASX J05481766-3239441, are $\alpha=05^{\mathrm{h}} 48^{\mathrm{m}} 25^{\mathrm{m}} .81, \delta=-32^{\circ} 39^{\prime} 49^{\prime} .5$, with no previously reported redshift in the literature. This edge-on anonymous galaxy lies almost aligned with the linear distribution of the AM 0546-324 system members. Is this object a candidate for tidal alignment? (see recent discussion on tidal alignment model of intrinsic galaxy alignments by Blazek et al. 2011).

The stellar formation history of 2MFGC 04711 and AM 0546-324 (NED02) galaxies were well represented by the stellar population synthesis code STARLIGHT (see Figs. 7 and 8). The synthesis results in flux fraction as a function of the distance to the center of each galaxy do not show any spatial variation in the contribution of the different stellar population components. Both galaxies are dominated by an old stellar population with age between $2 \times 10^{9}<t \leq 13 \times 10^{9} \mathrm{yr}$ in all apertures. 
Table 4. Stellar-population synthesis results.

\begin{tabular}{|c|c|c|c|c|c|c|c|c|c|c|}
\hline $\begin{array}{l}\text { Pos. } \\
(\operatorname{arcsec})\end{array}$ & $\begin{array}{c}x_{\mathrm{Y}} \\
\text { (per cent) }\end{array}$ & $\begin{array}{c}x_{\mathrm{I}} \\
\text { (per cent) }\end{array}$ & $\begin{array}{c}x_{\mathrm{O}} \\
\text { (per cent) }\end{array}$ & $\begin{array}{c}m_{\mathrm{Y}} \\
\text { (per cent) }\end{array}$ & $\begin{array}{c}m_{\mathrm{I}} \\
\text { (per cent) }\end{array}$ & $\begin{array}{c}m_{\mathrm{O}} \\
\text { (per cent) }\end{array}$ & $Z_{\star}{ }^{1}$ & $\chi^{2}$ & $\begin{array}{l}\text { Adev } \\
(\mathrm{mag})\end{array}$ & $A_{\mathrm{v}}$ \\
\hline \multicolumn{11}{|c|}{ 2MFGC 04711} \\
\hline-1.04 & 0.0 & 0.0 & 100.0 & 0.0 & 0.0 & 100.0 & 0.020 & 1.9 & 2.24 & 0.59 \\
\hline-0.90 & 0.0 & 0.0 & 100.0 & 0.0 & 0.0 & 100.0 & 0.027 & 1.9 & 1.24 & 0.07 \\
\hline-0.77 & 0.0 & 0.0 & 100.0 & 0.0 & 0.0 & 100.0 & 0.026 & 2.0 & 1.24 & 0.07 \\
\hline-0.65 & 0.0 & 0.0 & 100.0 & 0.0 & 0.0 & 100.0 & 0.027 & 1.9 & 1.25 & 0.07 \\
\hline-0.52 & 0.0 & 0.0 & 100.0 & 0.0 & 0.0 & 100.0 & 0.028 & 2.0 & 1.21 & 0.07 \\
\hline-0.39 & 0.0 & 0.0 & 100.0 & 0.0 & 0.0 & 100.0 & 0.027 & 2.0 & 1.24 & 0.07 \\
\hline-0.27 & 0.0 & 0.0 & 100.0 & 0.0 & 0.0 & 100.0 & 0.026 & 1.9 & 1.24 & 0.07 \\
\hline-0.14 & 0.0 & 0.0 & 100.0 & 0.0 & 0.0 & 100.0 & 0.028 & 2.0 & 1.22 & 0.07 \\
\hline-0.05 & 0.0 & 0.0 & 100.0 & 0.0 & 0.0 & 100.0 & 0.027 & 2.0 & 1.23 & 0.07 \\
\hline 0.0 & 0.0 & 0.0 & 100.0 & 0.0 & 0.0 & 100.0 & 0.023 & 1.8 & 1.30 & 0.00 \\
\hline 0.02 & 0.0 & 0.0 & 100.0 & 0.0 & 0.0 & 100.0 & 0.027 & 2.0 & 1.22 & 0.07 \\
\hline 0.11 & 0.0 & 0.0 & 100.0 & 0.0 & 0.0 & 100.0 & 0.027 & 2.0 & 1.22 & 0.07 \\
\hline 0.20 & 0.0 & 0.0 & 100.0 & 0.0 & 0.0 & 100.0 & 0.027 & 2.0 & 1.26 & 0.07 \\
\hline 0.30 & 0.0 & 0.0 & 100.0 & 0.0 & 0.0 & 100.0 & 0.027 & 1.9 & 1.24 & 0.07 \\
\hline 0.41 & 0.0 & 0.0 & 100.0 & 0.0 & 0.0 & 100.0 & 0.027 & 1.9 & 1.23 & 0.07 \\
\hline 0.54 & 0.0 & 0.0 & 100.0 & 0.0 & 0.0 & 100.0 & 0.027 & 1.9 & 1.24 & 0.07 \\
\hline 0.66 & 0.0 & 0.0 & 100.0 & 0.0 & 0.0 & 100.0 & 0.027 & 1.9 & 1.24 & 0.07 \\
\hline 0.79 & 0.0 & 0.0 & 100.0 & 0.0 & 0.0 & 100.0 & 0.027 & 1.9 & 1.23 & 0.07 \\
\hline 0.91 & 0.0 & 0.0 & 100.0 & 0.0 & 0.0 & 100.0 & 0.026 & 2.0 & 1.25 & 0.07 \\
\hline 1.06 & 0.0 & 15.0 & 85.0 & 0.0 & 4.9 & 95.1 & 0.041 & 1.9 & 1.93 & 0.00 \\
\hline 1.18 & 0.0 & 21.0 & 79.0 & 0.0 & 5.6 & 94.4 & 0.034 & 1.8 & 2.02 & 0.00 \\
\hline Integrated & 0.0 & 0.0 & 100.0 & 0.0 & 0.0 & 100.0 & 0.026 & 1.9 & 1.23 & 0.06 \\
\hline \multicolumn{11}{|c|}{ AM 0546-324 (NED02) } \\
\hline-0.68 & 0.0 & 0.0 & 100.0 & 0.0 & 0.0 & 100.0 & 0.026 & 2.2 & 1.41 & 0.02 \\
\hline-0.56 & 0.0 & 0.0 & 100.0 & 0.0 & 0.0 & 100.0 & 0.026 & 2.2 & 1.43 & 0.02 \\
\hline-0.44 & 0.0 & 0.0 & 100.0 & 0.0 & 0.0 & 100.0 & 0.025 & 2.2 & 1.36 & 0.02 \\
\hline-0.33 & 0.0 & 0.0 & 100.0 & 0.0 & 0.0 & 100.0 & 0.027 & 2.2 & 1.43 & 0.02 \\
\hline-0.21 & 0.0 & 0.0 & 100.0 & 0.0 & 0.0 & 100.0 & 0.027 & 2.2 & 1.41 & 0.02 \\
\hline-0.10 & 0.0 & 0.0 & 100.0 & 0.0 & 0.0 & 100.0 & 0.026 & 2.2 & 1.39 & 0.02 \\
\hline 0.00 & 0.0 & 0.0 & 100.0 & 0.0 & 0.0 & 100.0 & 0.025 & 2.2 & 1.24 & 0.02 \\
\hline 0.10 & 0.0 & 0.0 & 100.0 & 0.0 & 0.0 & 100.0 & 0.026 & 2.3 & 1.35 & 0.02 \\
\hline 0.21 & 0.0 & 0.0 & 100.0 & 0.0 & 0.0 & 100.0 & 0.026 & 2.2 & 1.34 & 0.02 \\
\hline 0.30 & 0.0 & 0.0 & 100.0 & 0.0 & 0.0 & 100.0 & 0.027 & 2.2 & 1.44 & 0.02 \\
\hline 0.40 & 0.0 & 0.0 & 100.0 & 0.0 & 0.0 & 100.0 & 0.026 & 2.2 & 1.43 & 0.02 \\
\hline 0.51 & 0.0 & 0.0 & 100.0 & 0.0 & 0.0 & 100.0 & 0.025 & 2.2 & 1.35 & 0.02 \\
\hline 0.63 & 0.0 & 0.0 & 100.0 & 0.0 & 0.0 & 100.0 & 0.025 & 2.2 & 1.42 & 0.02 \\
\hline 0.73 & 0.0 & 0.0 & 100.0 & 0.0 & 0.0 & 100.0 & 0.026 & 2.2 & 1.38 & 0.02 \\
\hline 0.84 & 0.0 & 0.0 & 100.0 & 0.0 & 0.0 & 100.0 & 0.024 & 2.4 & 1.63 & 0.17 \\
\hline Integrated & 0.0 & 0.0 & 100.0 & 0.0 & 0.0 & 100.0 & 0.020 & 1.7 & 1.54 & 0.00 \\
\hline
\end{tabular}

Notes. ${ }^{(1)}$ Abundance by mass with $Z_{\odot}=0.02$.

\section{Conclusions}

We reported optical band spectroscopy observations of the AM 0546-324 system, which is the core of Abell S0546 cluster of galaxies. Morphological substructures were found in an enhanced r-image of this system. This suggests that the members are presently undergoing early stages of tidal interaction.

Below is a summary of our main results:

- The AM0546-324 system is composed of four main galaxies: 2MFGC 04711, AM 0546-324 (NED02), the K galaxy, and the one named $\mathrm{S}$ galaxy by us. Adopting the $\mathrm{S}$ galaxy as the center of this gravitationally bound system, the radial velocity differences between the different quoted members vary from 43 to $646 \mathrm{~km} \mathrm{~s}^{-1}$.
- Within 1.2 arcmin of AM 0546-324 there are a few relevant field companions such as the $\mathrm{C}$ galaxy in the SE direction and a new Polar Ring galaxy candidate in the SW. Several dwarf objects in and surrounding this system are close enough to be candidate members of this system, but no quoted redshift for these objects was found in the literature.

- The S galaxy seems to be large enough to wrap up all principal companions with its smooth distribution of material.

- The spectra of 2MFGC 04711, NED02, S, and the C galaxy resemble those of early-type galaxies and no emission lines were detected. No star-forming regions and no nuclear ionization sources were detected in the observed regions of the four main galaxies.

- The calculated heliocentric radial velocity for the $S$ galaxy is $20141 \pm 10 \mathrm{~km} \mathrm{~s}^{-1}(z=0.0696)$, which agrees with the radial 


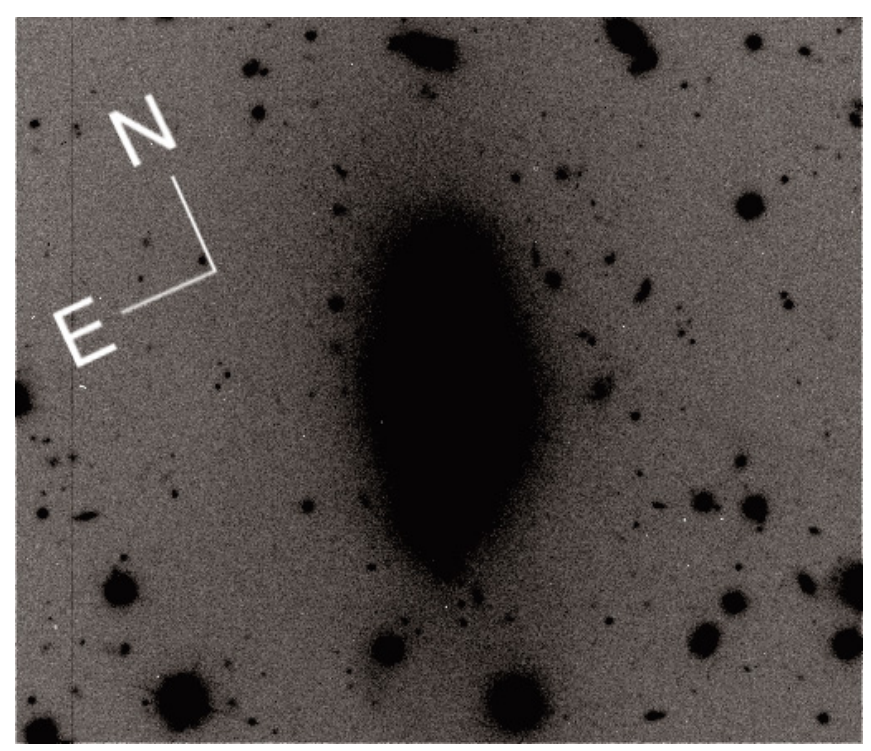

Fig. 9. Reproduction of the image of AM0546-324 with different contrast to highlight the dwarf objects crowding the system.

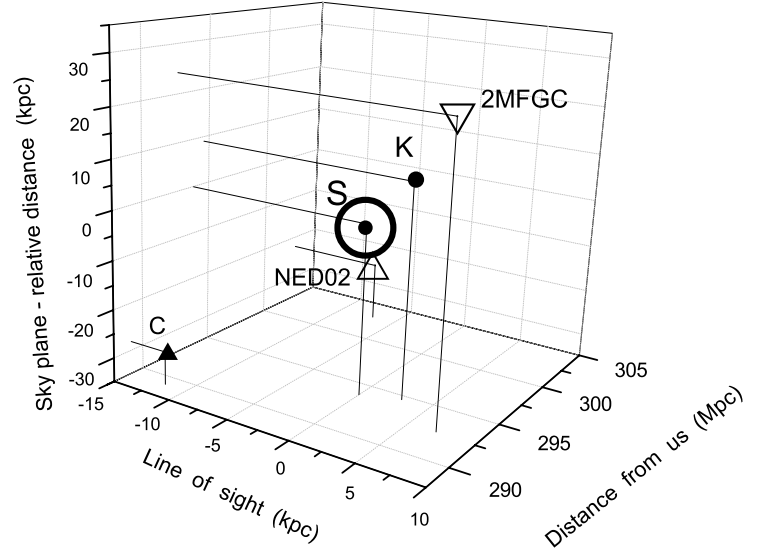

Fig. 10. Spatial distribution of the main galaxies of the AM 0546324 system centered on the Shadowy galaxy: the line-of-sight in kpc $(X$-axis); the calculated distance from us in Mpc ( $Y$-axis); and the relative distance between the objects in the sky-plane in kpc ( $Z$-axis). The $\mathrm{S}$ galaxy is displayed as a dot inside a circle.

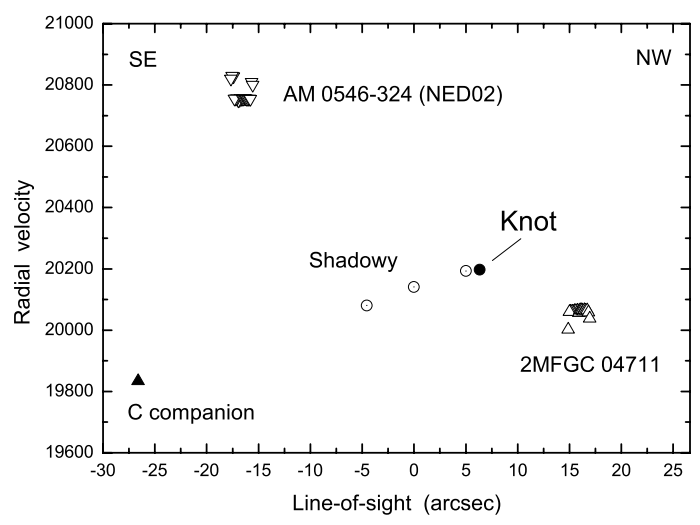

Fig. 11. Calculated radial velocity in $\mathrm{km} \mathrm{s}^{-1}$ versus the distribution of the main objects in the line-of-sight centered on the Shadowy galaxy. The open circles are the center, the SE and NW sections of the S galaxy, respectively.
Table 5. Radial velocity differences in the galaxy system.

\begin{tabular}{lc}
\hline \hline Pair & $\Delta V\left(\mathrm{~km} \mathrm{~s}^{-1}\right)$ \\
\hline Shadowy-2MFGC 04711 & 84 \\
Shadowy-Knot & 56 \\
Shadowy-NED02 & 613 \\
Shadowy-C galaxy & 307 \\
2MFGC 04711-Knot & 140 \\
2MFGC 04711-NED02 & 697 \\
NED02-C galaxy & 920 \\
\hline
\end{tabular}

velocity of the Abell S0546 cluster ( $c z=20893 \mathrm{~km} \mathrm{~s}^{-1}$ ); for $2 \mathrm{MFGC} 04711$, it is $20057 \pm 10 \mathrm{~km} \mathrm{~s}^{-1}(z=0.0693)$; and for NED02, it is $20754 \pm 10 \mathrm{~km} \mathrm{~s}^{-1}(z=0.0718)$, both in agreement with quoted values in NED.

- The C galaxy, $c z=19834 \pm 40 \mathrm{~km} \mathrm{~s}^{-1}(z=0.0685)$, and the $\mathrm{K}$ galaxy, $c z=20197(z=0.0698)$, are both bound members of the AM 0546-324 system.

- From the calculated mass lower limit, both 2MFGC 04711 and NED02 have $~ 31 \%$ of the mass of the S galaxy, and the C galaxy, almost $7 \%$.

- The rotation profiles of 2MFGC 04711 and NED02 are typical of tidal coupling in ellipticals with no net rotation, which results in a U-shaped rotation profile with the galaxy core at the base of the U. Both galaxies are gravitationally coupled directly with the proposed central object of the cluster, the $\mathrm{S}$ galaxy. The U-shaped structure is a direct observational signature of tidal friction with the extended material of the $\mathrm{S}$ galaxy.

- Internally, the no-net rotation core in the U-shaped rotation profile of both $2 \mathrm{MFGC} 04711$ and NED02 seems to be slightly perturbed by the tidal interaction with the $\mathrm{S}$ galaxy, which lies in the center of the local gravitational potential-well of this system.

- 2MFGC 04711 and AM 0546-324 (NED02) are completely dominated by an old stellar population with age between $2 \times$ $10^{9}<t \leq 13 \times 10^{9} \mathrm{yr}$.

In summary, AM 0546-324 is a system where signatures of tidal perturbations and friction are clearly visible. The deformity detected in the 2MFGC 04711, NED02, and K galaxies is due to large tidal forces exerted principally by the $\mathrm{S}$ galaxy (like the deformation and dynamical friction between two elliptical galaxies - Prugniel \& Combes 1992). Simultaneously, the S galaxy is perturbed by the whole interaction with all principal objects of the system.

Two questions still remain to be answered: (1) is the $\mathrm{S}$ galaxy environment the starting point for the birth of a future $\mathrm{cD}$ galaxy? and (2) what is the origin of the $\mathrm{S}$ galaxy?

Acknowledgements. This work was partially supported by the Ministerio da Ciência, Tecnologia e Inova cão (MCTI), Laboratório Nacional de Astrofísica, and Universidade do Vale do Paraíba - UNIVAP. A. C. Krabbe thanks the support of FAPESP, process 2010/01490-3. We also thank Ms. Alene Alder-Rangel and M. de Oliveira-Abans for editing the English in this manuscript. Based on observations obtained at the Gemini Observatory, which is operated by the Association of Universities for Research in Astronomy, Inc., under a cooperative agreement with the NSF on behalf of the Gemini partnership: the National Science Foundation (United States), the Science and Technology Facilities Council (United Kingdom), the National Research Council (Canada), CONICYT (Chile), the Australian Research Council (Australia), Ministerio da Ciência, Tecnologia e Inova cão (Brazil) and Ministerio de Ciencia, Tecnología e Innovación Productiva (Argentina). The observations were performed under the identification number GS-2010B-Q-7. This publication makes use of data products from the Two Micron All Sky Survey, which is a joint project of the University of Massachusetts and the Infrared Processing and Analysis 
Center/California Institute of Technology, funded by the National Aeronautics and Space Administration and the National Science Foundation. This research also used the NASA/IPAC Infrared Science Archive, which is operated by the Jet Propulsion Laboratory, California Institute of Technology, under contract with the National Aeronautics and Space Administration.

\section{References}

Abell, G. O. 1965, ARA\&A, 3, 1

Abell, G. O., Corwin Jr., H. G., \& Olowin, R. P. 1989, ApJS, 70, 1

Arp, H. C., \& Madore, B. F. 1977, Quart. J. Roy. Astron. Soc., 18, 234

Arp, H. C., \& Madore, B. F. 1986, Catalogue of Southern Peculiar Galaxies and

Associations, Vol. I, Positions and Descriptions (Toronto: Clarke-Irwin)

Asari, N. V., Cid Fernandes, R., Stasińska, G., et al. 2007, MNRAS, 381, 263

Astier, P., Guy, J., Regnault, N., et al. 2006, A\&A, 447, 31

Bacells, M., Borne, K. D., \& Hoessel, J. G. 1989, ApJ, 336, 655

Baldry, I. K., Glazebrook, K., Brinkmann, J., et al. 2004, ApJ, 600, 681

Barnes, J. E. 1992, AJ, 393, 484

Bender, R., Paquet, A., \& Nieto, J.-L. 1991, A\&A, 246, 349

Bezanson, R., van Dokkum, P. G., Franx, M., et al. 2011, ApJ, 737, L31

Blazek, J., McQuinn, M., \& Seljak, U. 2011, JCAP, 5, 010

Borne, K. D. 1988, ApJ, 330, 38

Borne, K. D. 1990, in Dynamics and Interactions of Galaxies, ed. R. Wielen (Berlin: Springer), 196

Borne, K. D., \& Hoessel, J. G. 1985, BAAS, 17, 601

Borne, K. D., \& Hoessel, J. G. 1988, ApJ, 330, 51

Borne, K. D., Balcells, M., Hoessel, J. G., \& McMaster, M. 1994, ApJ, 435, 79

Bournaud, F., Jog, C. J., \& Combes, F. 2005, A\&A, 437, 69

Bournaud, F., Jog, C. J., \& Combes F. 2007, A\&A, 476, 1179

Bruzual, G., \& Charlot, S. 2003, MNRAS, 344, 1000

Cid Fernandes, R., Gu, Q., Melnick, J., et al. 2004, MNRAS, 355, 273

Cid Fernandes, R., Mateus, A., Sodré, L., Stasińska, G., \& Gomes, J. M. 2005, MNRAS, 358, 363

Faber, S. M., Willmer, C. N. A., Wolf, C., et al. 2007, ApJ, 665, 265

Faúndez-Abans, M., \& de Oliveira-Abans, M. 1998a, A\&AS, 129, 357 (FAOA)

Faúndez-Abans, M., \& de Oliveira-Abans, M. 1998b, A\&AS, 128, 289

Faúndez-Abans, M., de Oliveira-Abans, M., Fernandes, I. F., et al. 2010, Proc.

Workshop OPD, SOAR and Gemini, Campos do Jordão, Brazil

Freedman, W. L., Madore, B. F., Gibson, B. K., et al. 2001, ApJ, 553, 47

Gerhard, O. E. 1993, MNRAS, 265, 213
Gordon, K. D., Clayton, G. C., Misselt, K. A., Landolt, A. U., \& Wolff, M. J. 2003, ApJ, 594, 279

Hernquist, L., \& Barnes, J. E. 1991, Nature, 354, 210

Hook, I. M., Jorgensen, I., Allington-Smith, J. R., et al. 2004, PASP, 166, 425

Kaviraj, S. 2010, MNRAS, 406, 382

Krabbe, A. C., Pastoriza, M. G., Winge, C., et al. 2011, MNRAS, 416, 38

Lang, K. R. 1999, Astrophysical Formulae, Vol. II Space, Time, Matter and Cosmology, 3rd edn. (Springer-Verlag)

Leeuwin, F., \& Combes, F. 1997, MNRAS, 284, 45

Lindegren, L., \& Dravins, D. 2003, A\&A, 401, 1185

Madejsky, R. 1991, A\&A, 247, 348

Madejsky, R., Bender, R., \& Möllenhoff, C. 1991, A\&A, 242, 58

Mauch, T., \& Sadler E. M. 2007, MNRAS, 375, 931

Mihos, J. C., Walker, I. R., Hernquist, L., Mendes de Oliveira, C., \& Bolte, M. 1995, ApJ, 447, 87

Mitronova, S. N., Karachentsev, I. D., Karachentseva, V. E., et al. 2004, Bull. S. Astro. Obs., 57, 5

Naab, T., \& Burkert, A. 2003, ApJ, 597, 893

Paturel, G., Vauglin, C., Petit, J., et al. 2005, A\&A, 430, 751

Pereira, M. J., \& Bryan, G. L. 2010, ApJ, 721, 939

Prugniel, Ph., \& Combes, F. 1992, A\&A, 259, 25

Quintana, H., \& Ramírez, A. 1995, A\&AS, 96, 343

Rogers, B., Ferreras, I., Kaviraj, S., Pasquali, A., \& Sarzi, M. 2009, MNRAS, 399, 2172

Salpeter, E. E. 1955, ApJ, 121, 161

Schafer, J. L. 1997, Analysis of incomplete multivariate data, book 72. Champan and Hall Series Monographs on Statistic and Applied Probability. Champan and Hall, London

Schlegel, D. J., Finkbeiner, D. P., \& Davis, M. 1998, ApJ, 500, 525

Spergel, D. N., Verde, L., Peiris, H. V., et al. 2003, ApJS, 148, 175

Springel, V. 2000, MNRAS, 312, 859

Toomre, A., \& Toomre, J. 1972, ApJ, 178, 623

van der Marel, R. P., \& Franx, M. 1993, ApJ, 407, 525

Weil, M. L., \& Hernquist, L. 1994, ApJ, 431, 79

Weil, M. L., \& Hernquist, L. 1996, ApJ, 460, 101

Wenderoth, E., Faúndez-Abans, M., Krabbe, A. C., de Oliveira-Abans M., \& Cuevas, H. 2011, A\&A, 529, A157

Wong, A. K. C., \& Chiu, D. K. Y. 1987, IEEE Transaction Pattern Analysis and Machine Intelligence, 9, 796

Wu, X., \& Barbara, D. 2002, SIGKDD Explorations, 4, 21 